\section{Dissociative hydrogen adsorption on palladium requires aggregates of three or more vacancies}

\author{
T. Mitsui ${ }^{\star}$, M. K. Rose ${ }^{\star} \dagger$, E. Fomin ${ }^{\star} \dagger$, D. F. Ogletree ${ }^{\star} \&$ M. Salmeron ${ }^{\star}$ \\ * Materials Sciences Division, Lawrence Berkeley National Laboratory, Berkeley, \\ California 94720, USA \\ $\dagger$ Department of Physics, University of California, Berkeley, California 94720, \\ USA
}

During reaction, a catalyst surface usually interacts with a constantly fluctuating mix of reactants, products, 'spectators' that do not participate in the reaction, and species that either promote or inhibit the activity of the catalyst. How molecules adsorb and dissociate under such dynamic conditions is often poorly understood. For example, the dissociative adsorption of the diatomic molecule $\mathrm{H}_{2}$-a central step in many industrially important catalytic processes-is generally assumed ${ }^{1}$ to require at least two adjacent and empty atomic adsorption sites (or vacancies). The creation of active sites for $\mathrm{H}_{2}$ dissociation will thus involve the formation of individual vacancies and their subsequent diffusion and aggregation ${ }^{2-6}$, with the coupling between these events determining the activity of the catalyst surface. But even though active sites are the central component of most reaction models, the processes controlling their formation, and hence the activity of a catalyst surface, have never been captured experimentally. Here we report scanning tunnelling microscopy observations of the transient formation of active sites for the dissociative adsorption of $\mathrm{H}_{2}$ molecules on a palladium (111) surface. We find, contrary to conventional thinking ${ }^{1}$, that two-vacancy sites seem inactive, and that aggregates of three or more hydrogen vacancies are required for efficient $\mathrm{H}_{2}$ dissociation.

The experiments were performed in an ultrahigh-vacuum (UHV) chamber with a total background pressure below $2 \times 10^{-10}$ torr and a hydrogen partial pressure of $5 \times 10^{-11}$ torr, as determined by mass spectrometry. The variable-temperature scanning tunnelling microscope (STM) apparatus ${ }^{7}$ was modified to cool the tip to the same temperature as the sample. The $\operatorname{Pd}(111)$ sample was prepared by cycles of noble-gas bombardment at $1,000 \mathrm{~K}$ with subsequent 'flashing' to $1,100 \mathrm{~K}$. Hydrogen was introduced via a leak valve.

The work presented here concerns the final stages of $\mathrm{H}_{2}$ adsorption and dissociation on the $\mathrm{Pd}(111)$ surface, when the $\mathrm{H}$ coverage is close to one monolayer (ML). In these conditions, the formation and dynamics of active sites can be observed most clearly. On the clean surface, hydrogen molecules dissociate readily at $37 \mathrm{~K}$, the lowest temperature of the present experiments. In agreement with earlier low-energy diffraction studies ${ }^{8}$ and total-energy calculations $s^{9,10}$, we have found by STM $^{11}$ that $\mathrm{H}$ atoms adsorb on the three-fold face-centred cubic (f.c.c.) sites. The $\mathrm{H}$ atoms appear as $15 \mathrm{pm}$ ( 1 picometre $=10^{-12} \mathrm{~m}$ ) depressions in the STM images, owing to reduced tunnelling probability (Fig. 1a). As the $\mathrm{H}$ coverage increases, an ordered structure with $\sqrt{ } 3 \times \sqrt{ } 3$ R30 periodicity relative to the substrate is formed at $1 / 3 \mathrm{ML}$, with one occupied and two empty f.c.c. sites per unit cell. This is followed, with further $\mathrm{H}$ adsorption, by another $\sqrt{3} \times \sqrt{3}$ structure at $2 / 3 \mathrm{ML}$ with two occupied and one empty f.c.c. sites per cell ${ }^{8,11}$. As the empty sites in this latter structure are not contiguous, it is perhaps not surprising that the sticking coefficient for dissociative adsorption of $\mathrm{H}_{2}$ drops substantially as the coverage approaches $2 / 3 \mathrm{ML}$. Above $50 \mathrm{~K}$ however, thermally activated diffusion produces vacancy-aggregates where $\mathrm{H}_{2}$ molecules can dissociate, allowing the adsorption process to continue up to the saturation value of $1 \mathrm{ML}$. The STM images of the surface close to $1 \mathrm{ML}$ coverage (Fig. $1 \mathrm{~b}$ ) reveal a $1 \times 1$ structure with a small, 5-pm corrugation (in contrast with the $15 \mathrm{pm}$ of the isolated $\mathrm{H}$ atoms), with almost all the f.c.c. sites occupied by $\mathrm{H}$ atoms. The surface contains defects, in the form of $\mathrm{H}$ vacancies (empty f.c.c. sites), which appear as bright protrusions in the images. The apparent height of these protrusions is $50 \mathrm{pm}$. Because of the 10-fold contrast difference the vacancy protrusions dominate the image contrast, and the $1 \times 1 \mathrm{H}$ areas appear rather dark (Fig. 1b).

The dynamics of vacancy diffusion and their annihilation by dissociative adsorption of $\mathrm{H}_{2}$ at a temperature of $65 \mathrm{~K}$ was followed by acquiring successive STM images of the same area at $75 \mathrm{~s}$ per
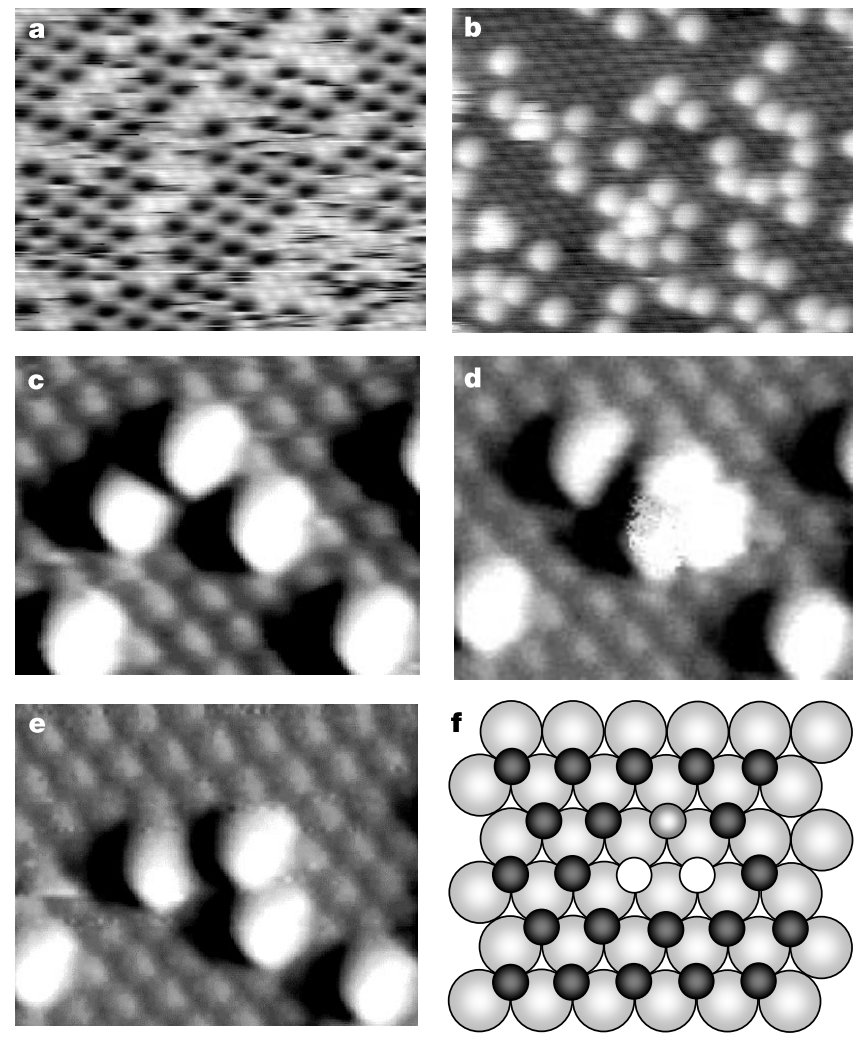

Figure 1 STM images of hydrogen atoms on $\mathrm{Pd}(111)$. a, $6.2 \times 5 \mathrm{~nm}$ at a coverage of $\sim 0.17 \mathrm{ML}$. The $\mathrm{H}$ atoms appear as dark spots (15-pm depressions) owing to reduced tunnelling current. They form islands with $\sqrt{ } 3 \times \sqrt{ } 3$ R30 periodicity. The bright spots between islands correspond to $\mathrm{Pd}$ atoms, and have a corrugation of $\sim 2 \mathrm{pm}$.

b, $6.4 \times 5.2 \mathrm{~nm}$ images acquired at a $\mathrm{H}$ coverage of almost $1 \mathrm{ML}$. The $\mathrm{H}$ atoms form an ordered $1 \times 1$ structure with weak, 5-pm corrugation. The large bright spots correspond to $\mathrm{H}$ vacancies, with an apparent height of about $50 \mathrm{pm}$. c-e, $2.0 \times 1.5 \mathrm{~nm}$ images, which are frames of a movie showing the formation and dissociation of a $\mathrm{H}$-vacancy pair in the almost $\mathrm{H}$-saturated $\mathrm{Pd}(111)$ surface. To enhance contrast, these images are shown shadowed from the right. In c, five $\mathrm{H}$ vacancies are present. A few minutes later (d), two vacancies have moved to nearest-neighbour positions forming a vacancy pair, which is imaged as a three-lobed triangle. In $\mathbf{e}$ the vacancies have separated, and five isolated vacancies can again be seen. The drawing in $\mathbf{f}$ illustrates the mechanism explaining the triangular appearance of the pair: large circles represent Pd atoms; small circles, $\mathrm{H}$ atoms. The light-shaded $\mathrm{H}$ atom can rapidly exchange with the vacancies (white circles) by hopping over bridge sites that offer the smallest barrier to diffusion. Exchange with the $\mathrm{H}$ atoms surrounding the triangle requires hopping near top Pd sites and/or close to other $\mathrm{H}$ atoms, a process with a higher barrier and therefore much slower. As the exchange is fast compared to the STM line-scan rate, each of the three triangle sites is vacant $2 / 3$ of the time and appears equally bright. Movies of Monte Carlo simulations of vacancy diffusion and aggregation can be viewed at http://stm.Ibl.gov (see also Supplementary Information). 

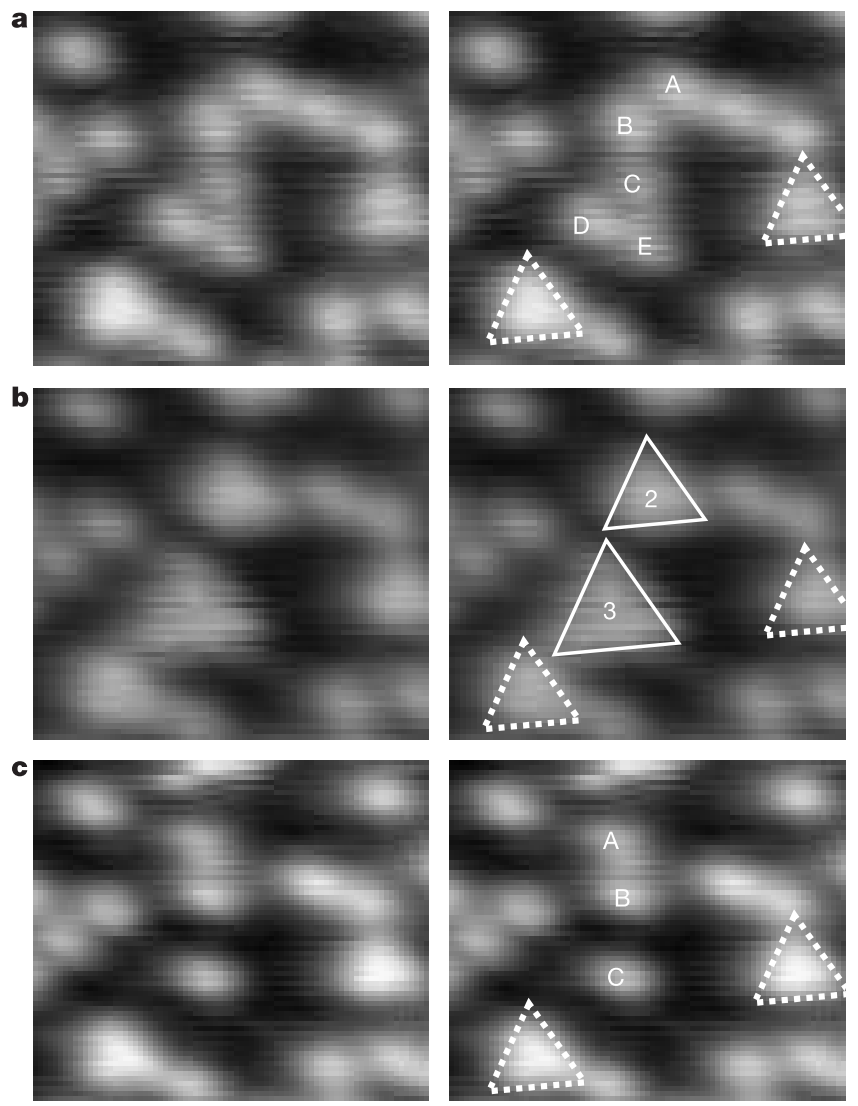

Figure 2 STM images from a movie showing the formation, separation and annihilation of $\mathrm{H}$-vacancy clusters. The contrast is such that only the $\mathrm{H}$ vacancies are visible in the form of bright spots. The images on the left $(3 \times 2.5 \mathrm{~nm})$ are repeated on the right with annotations. a, Five vacancies near the centre are labelled $(A-E)$ and two triangular vacancy pairs (2V) are marked with dashed triangles for reference. In $\mathbf{b}$, vacancies $A$ and $B$ have formed a $2 \mathrm{~V}$ cluster indicated by the triangle containing the number ' 2 '. Vacancies C-E have formed a six-site three-vacancy (3V) cluster, indicated by the larger triangle containing the number ' 3 '. Rapid motion of the $3 \mathrm{H}$ atoms inside the triangle explains the triangular appearance of these clusters. In $\mathbf{c}$ the $2 \mathrm{~V}$ pair has separated into isolated vacancies $A$ and $B$, while the $3 \mathrm{~V}$ cluster has been annihilated by dissociative adsorption of an $\mathrm{H}_{2}$ molecule, leaving a single remaining vacancy $\mathrm{C}$. The other $2 \mathrm{~V}$ clusters separated a few frames later.

frame. The gas-phase $\mathrm{H}_{2}$ pressure was increased to $2 \times 10^{-7}$ torr. At $65 \mathrm{~K}$ the observed vacancy hopping frequency was $4 \times 10^{-4} \mathrm{~s}^{-1}$, corresponding to an activation energy of $\sim 0.2 \mathrm{eV}$. For comparison, the activation energy for isolated $\mathrm{H}$-atom diffusion was found to be $\sim 0.09 \mathrm{eV}$ (ref. 11). Three frames from this experiment are shown (Fig. 1c-e). These images are shown with simulated 'shadows' from a light source on the right-hand side to enhance the contrast of the $1 \times 1 \mathrm{H}$ regions. In Fig. $1 \mathrm{c}$, five vacancies are visible. In the next frame (Fig. 1d) two of them, near the centre, have aggregated to form a vacancy pair, which later separates (Fig. 1e). Unexpectedly, the vacancy pair has the appearance of a bright triangle occupying three nearest-neighbour f.c.c. sites. When the pair separates, two isolated vacancies are again imaged.

The triangular shape of the vacancy pair can be explained with the help of the schematic diagram in Fig. 1f. According to total-energy calculations, the lowest-energy diffusion path for $\mathrm{H}$ is from an f.c.c. to an adjacent hexagonal close packed (h.c.p.) site across a bridge site, then across a second bridge to a neighbouring f.c.c. ${ }^{9}$. The lightshaded $\mathrm{H}$ atom in Fig. If can follow this path to either vacant site without hindrance, while all other surrounding $\mathrm{H}$ atoms (dark shaded in the diagram) must pass close to a neighbouring $\mathrm{H}$ atom
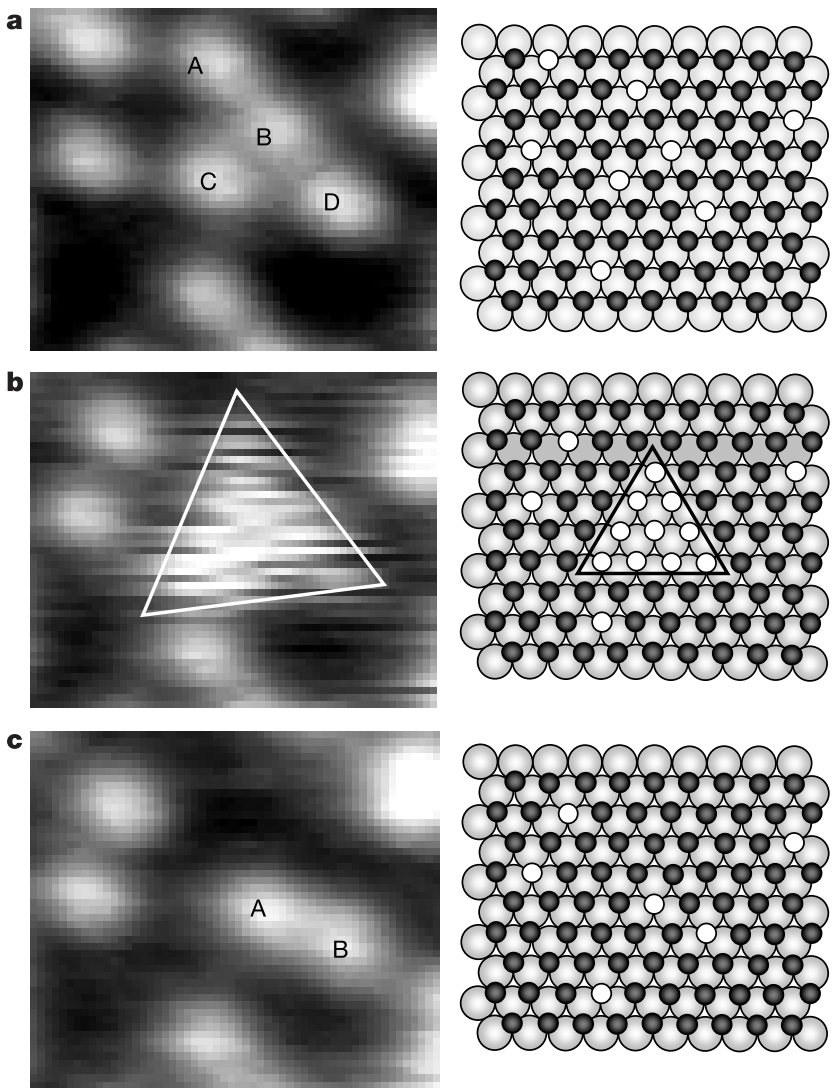

Figure 3 STM images from a movie showing the formation and annihilation of a four-vacancy (4V) cluster. The contrast is such that only the $\mathrm{H}$ vacancies are visible in the form of bright spots. The corresponding schematic diagrams show the $\mathrm{H}$ atoms and vacancies relative to the $\mathrm{Pd}(111)$ substrate. Letters $A-D$ mark four vacancies near the centre in $\mathbf{a}$. In $\mathbf{b}$, these vacancies have formed a ten-site $4 \mathrm{~V}$ cluster, indicated by the triangle, with $6 \mathrm{H}$ atoms rapidly moving inside. In $\mathbf{c}$ the $4 \mathrm{~V}$ cluster has been annihilated by adsorption of an $\mathrm{H}_{2}$ molecule, leaving two remaining vacancies.

(within 0.57 of the lattice spacing) or over a top site, a less favourable path. If the unhindered diffusion rate is fast compared to the STM imaging time, the light-shaded $\mathrm{H}$ atom will repeatedly change positions between the three corners of the triangular cluster, and the STM tip will 'see' each of the sites empty for $2 / 3$ of the time on average. As we shall see below this phenomenon is quite general, and occurs also with larger aggregates. Vacancy clusters always appear as partially occupied bright triangles bounded by closepacked $\{110\}$ edges, with $\mathrm{H}$ atoms diffusing rapidly inside.

Events where more than two vacancies aggregate to form larger clusters were also observed (Fig. 2). In Fig. 2a we can see several isolated vacancies and two vacancy pairs. In Fig. $2 \mathrm{~b}$ the five labelled vacancies of Fig. 2a have formed two new clusters: a vacancy pair $(2 \mathrm{~V})$ and a triplet $(3 \mathrm{~V})$. The $3 \mathrm{~V}$ cluster occupies a six-site triangular area bounded by $\{110\}$ edges, similar to the $2 \mathrm{~V}$ case, with three $\mathrm{H}$

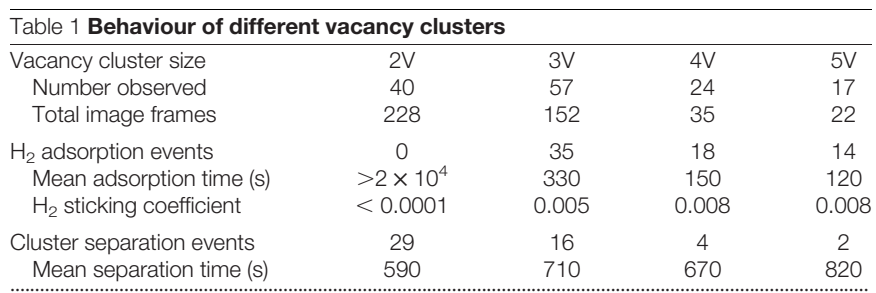

Active site statistics for vacancy clusters at $65 \mathrm{~K}$ in $2 \times 10^{-7}$ torr $\mathrm{H}_{2}$. STM frame time, $75 \mathrm{~s}$. $\mathrm{H}_{2}$ sticking coefficient estimated by assuming an $\mathrm{H}_{2}$ capture cross-section corresponding to the geometrical area of the $\mathrm{Pd}(111)$ unit cell per $\mathrm{H}$ vacancy. In addition to adsorption or separation, some clusters changed size by merging with additional vacancies. 


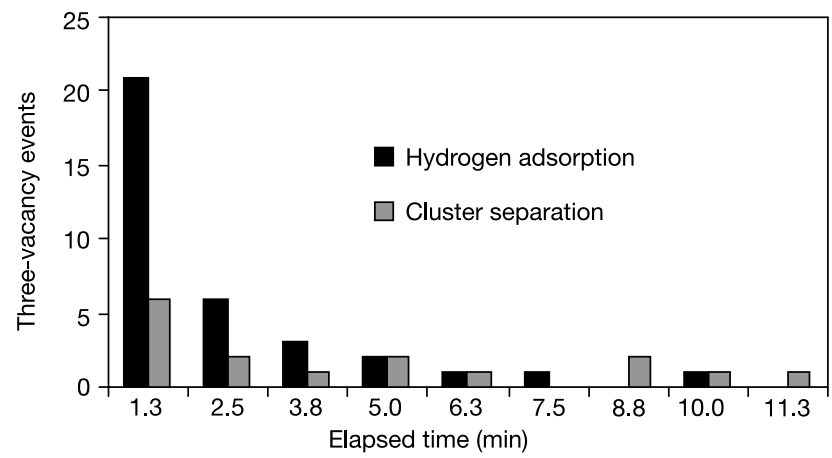

Figure $\mathbf{4}$ Lifetimes of three-vacancy clusters. Shown is the observed lifetime distribution of three-vacancy (3V) clusters at $65 \mathrm{~K}$ in a background pressure of $\sim 2 \times 10^{-7}$ torr $\mathrm{H}_{2}$. The clusters decay either by separation or by annihilation following adsorption of a $\mathrm{H}_{2}$ molecule, which fills two of the three $\mathrm{H}$ vacancies.

atoms diffusing rapidly among the six sites. In contrast to the $2 \mathrm{~V}$ clusters, the larger $3 \mathrm{~V}$ clusters are active for $\mathrm{H}_{2}$ dissociation (Fig. 2c). The $2 \mathrm{~V}$ cluster has separated into isolated vacancies (A, B), while the $3 \mathrm{~V}$ cluster has been annihilated by a dissociating $\mathrm{H}_{2}$ molecule, leaving one vacancy $(\mathrm{C})$ behind. Two other $2 \mathrm{~V}$ clusters have remained intact during this process, but separate several frames later (not shown). The annihilation of $3 \mathrm{~V}$ clusters occurs only in the presence of $\mathrm{H}_{2}$ background gas.

As a final example, Fig. 3 shows the formation and annihilation of a $4 \mathrm{~V}$ cluster. Again, this cluster appears as a triangle bounded by $\{110\}$ edges and occupying an area containing ten Pd atoms. The six $\mathrm{H}$ atoms in the triangle diffuse rapidly among the ten sites. This cluster decayed via $\mathrm{H}_{2}$ adsorption, leaving a pair of isolated vacancies. For this and for larger vacancy clusters, annihilation by $\mathrm{H}_{2}$ adsorption was the dominant decay mechanism.

Figure 4 shows the observed lifetime distribution for $3 \mathrm{~V}$ clusters in $2 \times 10^{-7}$ torr of $\mathrm{H}_{2}$, and Table 1 gives statistics for $2 \mathrm{~V}$ to $5 \mathrm{~V}$ clusters. Hydrogen adsorption was frequently observed for $3 \mathrm{~V}$ and larger clusters, always reducing the number of vacancies by two. The $\mathrm{H}_{2}$ sticking probability can be estimated at $0.5-0.8 \%$ for $3 \mathrm{~V}$ and larger clusters. In contrast, we never observed $\mathrm{H}_{2}$ adsorption for a $2 \mathrm{~V}$ cluster even though two atomic hydrogen sites are available. This finding is contrary to accepted models of diatomic molecule dissociation, and calls for a theoretical effort in modelling the adsorption step in catalysis that includes the precise atomic structure of the target sites and the role of adsorbate diffusivity in generating such sites.

As a first step to understanding the dynamics of vacancy aggregation, we performed Monte Carlo simulations of vacancy diffusion. The model, which uses only experimentally estimated values of the diffusion barriers of $\mathrm{H}$ atoms and vacancies (ref. 11; see also http:// stm.lbl.gov), correctly reproduced the observed stability and the triangular appearance of the $2 \mathrm{~V}, 3 \mathrm{~V}$ and $4 \mathrm{~V}$ clusters, and could thus help to improve our molecular-level understanding of the formation of the active sites that determine the activity of catalytically active surfaces.

Received 13 January; accepted 4 March 2003; doi:10.1038/nature01557.

1. Conrad, H., Ertl, G. \& Latta, E. E. Adsorption of hydrogen on palladium single crystal surfaces. Surf. Sci. 41, 435-446 (1974).

2. Taylor, H. S. Theory of the catalytic surface. Proc. R. Soc. Lond. A 108, 105-111 (1925).

3. Boudart, M. Four decades of active centers. Am. Sci. 57, 97-111 (1969).

4. Ponec, V. \& Sachtler, W. M. H. The reactions between cyclopentane and deuterium on nickel and nickel-copper alloys. J. Catal. 24, 250-261 (1972).

5. Sinfelt, J. H. Bimetallic Catalysts: Discoveries, Concepts and Applications (Wiley and Sons, New York, 1983).

6. Somorjai, G. A. Introduction to Surface Chemistry and Catalysis (Wiley and Sons, New York, 1994).

7. Behler, S. et al. A scanning tunneling microscope with continuous flow cryostat sample cooling. Rev. Sci. Instrum. 68, 2479-2485 (1997).

8. Felter, T. E., Sowa, E. C. \& Van Hove, M. A. Location of hydrogen on palladium (111) studied by lowenergy electron diffraction. Phys. Rev. B 40, 891-899 (1989).
9. Paul, J. F. \& Sautet, P. Density-functional periodic study of the adsorption of hydrogen on a palladium (111) surface. Phys. Rev. B 53, 8015-8027 (1996).

10. Lovvik, O. M. \& Olsen, R. A. Adsorption energies and ordered structures of hydrogen on $\operatorname{Pd}(111)$ from density functional periodic calculations. Phys. Rev. B 58, 10890-10898 (1998).

11. Mitsui, T., Rose, M. K., Fomin, E., Ogletree, D. F. \& Salmeron, M. Hydrogen adsorption and diffusion on Pd(111). Surf. Sci. (submitted).

Supplementary Information accompanies the paper on Nature's website

( http://www.nature.com/nature).

Acknowledgements This work was supported by the Office of Basic Energy Science of the US Department of Energy.

Competing interests statement The authors declare that they have no competing financial interests.

Correspondence and requests for materials should be addressed to M.S.

(e-mail: salmeron@stm.lbl.gov)

\section{Global anisotropy and the thickness of continents}

Yuancheng Gung, Mark Panning \& Barbara Romanowicz

Berkeley Seismological Laboratory and Department of Earth and Planetary Science, Berkeley, California 94720, USA

For decades there has been a vigorous debate about the depth extent of continental roots ${ }^{1,2}$. The analysis of heat-flow ${ }^{3}$, mantlexenolith $^{4}$ and electrical-conductivity ${ }^{5}$ data all indicate that the coherent, conductive part of continental roots (the 'tectosphere') is at most $200-250 \mathrm{~km}$ thick. Some global seismic tomographic models agree with this estimate, but others suggest that a much thicker zone of high velocities lies beneath continental shields ${ }^{6-9}$, reaching a depth of at least $400 \mathrm{~km}$. Here we show that this disagreement can be reconciled by taking into account seismic anisotropy. We show that significant radial anisotropy, with horizontally polarized shear waves travelling faster than those that are vertically polarized, is present under most cratons in the depth range $250-400 \mathrm{~km}$-similar to that found under ocean basins $\mathrm{s}^{9,10}$ at shallower depths of $80-250 \mathrm{~km}$. We propose that, in both cases, the anisotropy is related to shear in a low-viscosity asthenospheric channel, located at different depths under continents and oceans. The seismically defined 'tectosphere' is then at most $200-250 \mathrm{~km}$ thick under old continents. The 'Lehmann discontinuity', observed mostly under continents at about 200$250 \mathrm{~km}$, and the 'Gutenberg discontinuity', observed under oceans at depths of about $60-80 \mathrm{~km}$, may both be associated with the bottom of the lithosphere, marking a transition to flowinduced asthenospheric anisotropy.

The maximum thickness of the lithosphere, defined as a region of distinctly faster than average seismic velocities $(1.5 \%-2 \%)$ in global $S$-wave velocity $V_{\mathrm{S}}$ tomographic models, ranges from 200 to $400 \mathrm{~km}$, depending on the model ${ }^{6-9}$. This is clear from the drop in correlation between some models from around 0.80 at $100 \mathrm{~km}$ to less than 0.45 at $300 \mathrm{~km}$ depth (Fig. 1a), which casts some doubt on the ability of global tomography to accurately resolve upper mantle structure. However, although global $V_{\mathrm{S}}$ models differ from each other significantly in the depth range $200-400 \mathrm{~km}$ under the main continental shields, these differences are consistent when they are classified into three categories, depending on the type of data used to derive them: 'SV' (mostly vertical or longitudinal component data, dominated by Rayleigh waves in the upper mantle), ' $\mathrm{SH}$ ' (mostly transverse component data, dominated by Love waves), and 'hybrid' (three-component data). SH and hybrid models are better 